\title{
Fenótipo de fragilidade: influência de cada item na determinação da fragilidade em idosos comunitários - Rede Fibra
}

\author{
Phenotype of frailty: the influence of each item in determining \\ frailty in community-dwelling elderly - The Fibra Study
}

Silvia Lanziotti Azevedo da Silva ${ }^{1}$

Anita Liberalesso Neri ${ }^{2}$

Eduardo Ferrioli ${ }^{3}$

Roberto Alves Lourenço ${ }^{4}$

Rosângela Corrêa Dias ${ }^{5}$

${ }^{1}$ Escola de Enfermagem, Curso de Fisioterapia, Universidade Federal de Alfenas. R. Gabriel Monteiro da Silva 700, Centro. 37130000 Alfenas MG Brasil. silviafisiojf@yahoo.com.br ${ }^{2}$ Departamento de Psicologia Educacional, Faculdade de Educação, Universidade Estadual de Campinas. Campinas SP Brasil.

${ }^{3}$ Universidade de São Paulo. São Paulo SP Brasil.

${ }^{4}$ Policlínica Piquet Carneiro, Universidade do Estado do Rio de Janeiro. Rio de Janeiro RJ Brasil.

${ }^{5}$ Departamento de

Fisioterapia e Terapia

Ocupacional, Escola

de Educação Física,

Universidade Federal de

Minas. Belo Horizonte MG

Brasil.
Abstract The phenotype of frailty is used to assess frailty among the elderly by examining the following items: weight loss; exhaustion; low level of physical activity; weakness; and slow gait speed. The aim of the study was to evaluate the contribution of each item to determine the frailty syndrome among elderly Brazilians. The analysis was done using Multinomial Logistic Regression. The total sample of 5532 randomly selected elderly people in many cities in Brazil between December 2008 and September 2009 was assessed using the phenotype of frailty. The most frequent items were level of physical activity, followed by muscular weakness and slow gait speed. Items that were more likely to develop frailty, when positive, were slow gait speed $(O R=10.50,95 \% C I 8.55-12.90, p<0.001)$ and muscular weakness $(O R=7.31,95 \% C I$ 6,02 $8,86, p<0.001)$. The final model with five items explained $99.6 \%$ of frailty in the sample. These results suggested that the level of physical activity, weakness and slow gait speed were the items that most influence the determination of frailty, however the application of all items of the phenotype of frailty is the best way to assess frailty.

Key words Frail elderly, Aging, Epidemiology, Odds ratio
Resumo O fenótipo de fragilidade é uma ferramenta utilizada para avaliação da fragilidade em idosos, composto pelos itens perda de peso, exaustão, baixo nível de atividade física, fraqueza muscular e lentidão na marcha. O objetivo deste estudo foi avaliar a participação de cada item na determinação da fragilidade em idosos brasileiros. A análise foi feita pela Regressão Logística Multinomial. A amostra total de 5532 idosos, selecionados aleatoriamente em diversas cidades brasileiras entre dezembro de 2008 e setembro de 2009, foi avaliada pelo fenótipo de fragilidade. Os itens mais frequentes na amostra foram o nível de atividade física, seguido da fraqueza muscular $e$ lentidão da marcha. Os itens que apresentaram maior chance para o desenvolvimento da fragilidade foram a lentidão na marcha $(O R=10,50$, IC95\%8,55-12,90, $p<0,001)$ e a fraqueza mus-

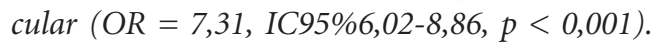
O modelo com os cinco itens explicou $99,6 \%$ da fragilidade na amostra. Tais resultados sugerem que o nível de atividade física, fraqueza muscular e lentidão na marcha são os itens que mais influenciam na determinação da fragilidade, mas a aplicação de todos os itens do fenótipo em conjunto é a melhor forma para a avaliação.

Palavras-chave Idoso Fragilizado, Envelhecimento, Epidemiologia, Razão de chances 


\section{Introdução}

A Síndrome da Fragilidade é resultado de deficiências multissistêmicas, que geram alterações diferenciadas do processo normal de envelhecimento ${ }^{1}$. Fried et al. $^{2}$ definem fragilidade como queda da reserva e resistência a estressores do organismo, levando a redução da capacidade de retorno à homeostase. Os componentes físicos da fragilidade são destacados neste conceito e interagem em forma de ciclo, o da fragilidade, e estão apoiados no tripé sarcopenia, desregulação neuroendócrina e alterações imunológicas ${ }^{2,3}$.

O fenótipo de fragilidade, proposta operacional de Fried et al. ${ }^{2}$, é capaz de traduzir as manifestações do ciclo da fragilidade ${ }^{2,3}$. É composto pelos itens perda de peso, exaustão, nível de atividade física, força muscular e lentidão da marcha ${ }^{2}$, e classifica o idoso em frágil, pré-frágil e não frágil, de acordo com número de itens positivos ${ }^{2}$.

O fenótipo, mesmo não sendo "padrão-ouro" para avaliação da síndrome de fragilidade, tem validade concorrente satisfatória, evidenciada pela associação com idade avançada, condições crônicas, função cognitiva e sintomas depressivos e pelo valor preditivo para desfechos adversos como quedas, hospitalização, incapacidade e morte $^{4-6}$.

Em relação à contribuição de cada item do fenótipo na determinação da fragilidade, Drey et al. ${ }^{7}$ encontraram como o mais comum a exaustão e o menos comum o baixo nível de atividade física. Já Rothman et al. ${ }^{8}$ encontraram os itens mais comuns a lentidão da marcha e o nível de atividade física, sendo que o nível de atividade física foi o que mais aumentou no acompanhamento da amostra.

Na população brasileira, um estudo encontrou o item lentidão da marcha como o mais frequente, tanto em idosos frágeis como préfrágeis ${ }^{9}$, e em outro estudo o mais comum foi a exaustão, seguida pela lentidão da marcha e força muscular ${ }^{10}$.

Apesar destes dados, ainda não existem estudos com grandes amostras da população brasileira acerca da contribuição individual e em conjunto com os outros de cada item na determinação da síndrome da fragilidade. Na prática clínica dos profissionais que lidam com idosos, em todos os níveis de atenção, avaliar os itens do fenótipo e determinar quais necessitam de maior atenção para abordagem precoce, pode ajudar na prevenção do avanço da fragilidade e suas conse- quências adversas para a saúde. Assim, o objetivo deste estudo foi determinar o quanto cada item do fenótipo de fragilidade contribui para a determinação da síndrome, sozinho ou em conjunto com os demais em uma amostra de base populacional de idosos brasileiros.

\section{Métodos}

A Rede FIBRA (Perfis de Fragilidade de Idosos Brasileiros) é um estudo observacional de caráter transversal de base populacional, multicêntrico e multidisciplinar, realizado em diversas cidades brasileiras, com diferentes Índices de Desenvolvimento Humano (IDH). Na amostra do presente estudo fizeram parte idosos comunitários das seguintes cidades: Barueri (SP), Belém (PA), Belo Horizonte (MG), Campinas (SP), Cuiabá (MT), Ermelindo Matarazzo (SP), Fortaleza (CE), Ivotí (RS), Juiz de Fora (MG), Parnaíba (PI), Poços de Caldas (MG), Recife (PE), Ribeirão Preto (SP) e Santa Cruz (RN).

Em cada cidade, a amostra foi calculada com base em cálculo amostral, para garantia da representatividade populacional local, e a coleta foi realizada em setores censitários diferenciados, que variavam de acordo com a concentração de idosos, e, portanto, também no número dos incluídos em cada setor. O número total de idosos variou conforme a cidade, pois a amostra por conglomerado gerou diferenças pela população total e percentual deles em cada local.

Foram sorteadas ruas em cada setor determinado e nelas foram visitadas todas as casas em busca de indivíduos com mais de 65 anos, residentes na comunidade, que concordassem em participar da entrevista e de testes físicos pertencentes ao instrumento, aplicados por entrevistadores previamente treinados. As coletas ocorreram entre dezembro de 2008 e dezembro de 2009.

Os critérios de exclusão foram sequelas graves de Acidente Vascular Encefálico (AVE), doenças neurológicas que impedissem a realização dos testes, usar cadeira de rodas ou estar acamado e apresentar déficit cognitivo indicado pela pontuação inferior à 17 no Mini-Exame do Estado Mental (MEEM) ${ }^{11}$.

O projeto foi aprovado pelo Comitê de Ética em Pesquisa e todos os participantes assinaram Termo de Consentimento Livre e Esclarecido (TCLE). 


\section{Caracterização da amostra}

Para caracterização da amostra foram consideradas as variáveis idade, sexo, estado civil, cor/ raça, número de comorbidades avaliado por autorrelato de diagnóstico médico no ano anterior de hipertensão, diabetes, doenças pulmonares e cardíacas, osteoartrite e osteoporose, autorrelato da ocorrência de quedas no ano anterior, capacidade funcional para Atividades Básicas pela Escala de Katz ${ }^{12}$, Instrumentais pela escala de Lawton $^{13}$ e Avançadas de Vida Diária pela escala Multidemensional Health Assessment Quiestionaire ${ }^{14}$ adaptada, medo de cair avaliado pela Falls Efficacy Scale International - Brasil ${ }^{15}$ e indicativo de depressão pela Geriatric Depression Scale Brasil $^{16}$. Cada uma dessas escalas gerou em escore total que foi comparado entre os grupos de idosos frágeis, pré-frágeis e não frágeis.

\section{Avaliação de Fragilidade}

Pela aplicação do fenótipo de fragilidade ${ }^{2}$, esta foi avaliada bem como seus pontos de corte originais, segundo os itens:

(1) Perda de peso: autorrelato de perda de peso não intencional no último ano superior à $4 \mathrm{~kg}$ considerado positivo $^{2}$;

(2) Exaustão: questões 7 e 20 da escala Center of Epidemiological Scale - Depression $(C E S-D)^{17}$, respostas "na maioria das vezes" e "sempre" para pelo menos uma das perguntas pontua positivo para este critério;

(3) Nível de Atividade Física: gasto calórico fornecido pela aplicação do questionário $M i$ nessota Leisure Time Activity ${ }^{18}$, sendo positivo em casos de valores inferiores a pontos de corte ajustados por sexo; para mulheres, foram consideradas frágeis com gasto $<270 \mathrm{kcal}$ por semana, e homens com gasto $<383 \mathrm{kcal}$ por semana ${ }^{2}$

(4) Diminuição da força muscular: valor em kilograma-força (kgf) obtido pelo teste com o dinamômetro de preensão palmar Jamar ${ }^{\circledR}$, positivo em casos de valores inferiores a ponto de corte ajustado por sexo e Índice de Massa Corporal $(\mathrm{IMC})^{2}$; para mulheres, foram consideradas frágeis aquelas com força $<17($ IMC $<23),<17.3$ (IMC 23.1 - 26),$<18$ (IMC 26.1 - 29) e $<21$ (IMC >29); para os homens, foram considerados frágeis aqueles com força $<29($ IMC $<24),<30$ $($ IMC $24.1-28)$ e $<32(\text { IMC }>28)^{2}$.

(5) Lentidão na marcha: valor do tempo, em segundos (s), gasto para percorrer 4,6 metros (m) em um total de $8,6 \mathrm{~m}$, descontando $2 \mathrm{~m}$ iniciais e finais de aceleração e desaceleração, sendo positi- vos valores superiores a ponto de corte ajustado por sexo e altura ${ }^{2}$. Para mulheres, foram consideradas frágeis aquelas que demoraram mais que 7 segundos $(<159 \mathrm{~cm})$ ou 6 segundos $(>160 \mathrm{~cm})$, homens foram consideradas frágeis quando demoraram mais que 7 segundos $(<173 \mathrm{~cm})$ ou 6 segundos $(<173 \mathrm{~cm})$.

Foram considerados frágeis idosos que pontuaram positivo em 3, 4 ou 5 itens, pré-frágeis aqueles que pontuaram positivo em 1 ou 2 itens e não frágeis os que não pontuarem em nenhum item.

\section{Analise estatística}

A descrição da amostra foi feita por medidas de média e desvio-padrão para variáveis numéricas e percentuais para variáveis categóricas. Diferenças entre os valores de tais variáveis entre idosos frágeis, pré-frágeis e não frágeis foram avaliados por ANOVA com pós-teste de Tukey, em casos de variáveis numéricas, e qui-quadrado em casos de categóricas. Todas as variáveis tiveram distribuição normal, avaliada pelo teste de Kolmogorov-Smirnov, o que justifica o uso da estatística paramétrica.

Dentro da análise descritiva, foi calculada a frequência de cada um dos itens do fenótipo de fragilidade na amostra do estudo.

A avaliação da influencia de cada item do fenótipo foi feita por análise de Regressão Logística Multinomial. Foram feitos 5 modelos de cada item individualmente na classificação de fragilidade final, sendo a variável dependente capaz de assumir os valores frágil ou pré-frágil.

Foram traçados 3 modelos de Regressão Logística Multinomial para combinações de itens, sendo um feito com o agrupamento de perda de peso e fadiga, outro agrupando baixo nível de atividade física, força muscular e lentidão da marcha, e, por fim, um modelo que agrupasse os cinco itens em conjunto.

As análises foram feitas no programa estatístico SPSS 17.0 e foi considerado nível de significância $\alpha=0,05$.

\section{Resultados}

A amostra total foi composta por 5532 idosos comunitários de várias cidades brasileiras. A maioria da amostra eram mulheres $(65,6 \%)$ e a média de idade foi de 73,08 $( \pm 6,17)$ anos. As características da amostra estão relatadas na Tabela 1.

Todas as características sociodemográficas da amostra, exceto cor/raça, foram diferentes entre 
os três grupos de fragilidade. Em relação ao número de quedas, a diferença ocorreu apenas entre frágeis e não frágeis (Tabela 1).

A hipertensão arterial sistêmica foi a comorbidade mais relatada entre os idosos, seguida pela osteoartrite, independente do nível de fragilidade. O percentual das comorbidades foi diferente entre os grupos de fragilidade para doenças do coração, acidente vascular encefálico, diabetes mellitus, osteoartrite, depressão e osteoporose (Tabela 2).

Em relação à frequência de cada item de fragilidade, o baixo nível de atividade física foi o item mais comum na amostra total $(27,5 \%)$, entre os idosos pré-frágeis $(36,7 \%)$ e entre idosos frágeis $(78,2 \%)$. Entre os idosos frágeis, a lentidão na marcha também foi bastante frequente, positivo em 76,7\% deste grupo. O observação dos valores da frequência de todos os cinco itens foi maior entre os idosos frágeis. (Tabela 3 ).

Os itens foram associados de forma diferenciada em relação à condição de fragilidade, uma vez que os idosos já eram pré-frágeis, possuindo 1 ou 2 itens positivos. Baixo nível de atividade física $\left(\mathrm{R}^{2}=0,37\right)$, lentidão da marcha $\left(\mathrm{R}^{2}=0,32\right)$ e força muscular $\left(R^{2}=0,28\right)$ foram os itens que, isoladamente, tiveram maior poder de explicação para a fragilidade, dentro de modelos individuais. Idosos positivos para lentidão da marcha (OR $=10,50$ IC95\%8,55-12,90, $\mathrm{p}<0,001)$ tiveram maior chance de se tornaram frágeis (Tabela 4).

Foram gerados modelos alternativos com os itens com maior valor individual e menor valor individual da odds ratio (OR). O modelo que continha o baixo nível de atividade física, força muscular e lentidão da marcha explicou $69,6 \%$ da instalação da fragilidade, sendo que a redução da força muscular foi o item que, quando positivo, determinou a maior chance do idoso se tornar frágil, já sendo pré-frágil $(\mathrm{OR}=18,11$, IC95\%13,65-24,02, p < 0,001) (Tabela 5).

A combinação dos itens exaustão e perda de peso foram capazes de explicar, juntos, $39 \%$ da fragilidade em idosos pré-frágeis. Neste modelo,

\begin{tabular}{|c|c|c|c|c|c|}
\hline & $\begin{array}{c}\text { Amostra total } \\
\mathrm{n}=5532\end{array}$ & $\begin{array}{l}\text { Não frágeis } \\
\mathrm{n}=2094\end{array}$ & $\begin{array}{l}\text { Pré-frágeis } \\
n=2821\end{array}$ & $\begin{array}{l}\text { Frágeis } \\
n=617\end{array}$ & $\mathbf{p}$ \\
\hline Idade & $73,08( \pm 6,17)$ & $(37,8 \%)$ & $(51 \%)$ & $(11,2 \%)$ & $0,000^{*}$ \\
\hline Sexo & & $71,81( \pm 5,42)$ & $73,24( \pm 6,15)$ & $76,70( \pm 7,09)$ & $0,000^{* *}$ \\
\hline Masculino & $34,4 \%$ & $36,5 \%$ & $34 \%$ & $27,7 \%$ & \\
\hline Feminino & $65,6 \%$ & $63,5 \%$ & $66 \%$ & $72,3 \%$ & \\
\hline Estado civil & & & & & $0,000^{* *}$ \\
\hline Solteiro & $9,6 \%$ & $8,1 \%$ & $10,1 \%$ & $11,9 \%$ & \\
\hline Casado & $49,7 \%$ & $55,0 \%$ & $48,2 \%$ & $38,2 \%$ & \\
\hline Divorciado & $6,6 \%$ & $7,5 \%$ & $6,0 \%$ & $6,3 \%$ & \\
\hline Viúvo & $33,9 \%$ & $29,3 \%$ & $35,6 \%$ & $43,5 \%$ & \\
\hline Cor/Raça & & & & & 0,154 \\
\hline Branca & $52,3 \%$ & $54,0 \%$ & $52,0 \%$ & $50,8 \%$ & \\
\hline Negra & $9,5 \%$ & $8,8 \%$ & $10,0 \%$ & $8,8 \%$ & \\
\hline Mulata & $35,6 \%$ & $34,6 \%$ & $35,9 \%$ & $39,5 \%$ & \\
\hline Indígena & $0,7 \%$ & $0,6 \%$ & $0,8 \%$ & $0,4 \%$ & \\
\hline Oriental & $1,3 \%$ & $1,4 \%$ & $1,4 \%$ & $0,5 \%$ & \\
\hline Número de comorbidades & $1,86( \pm 1,44)$ & $1,69( \pm 1,33)$ & $1,92( \pm 1,47)$ & $2,55( \pm 1,58)$ & $0,000^{*}$ \\
\hline Sofreu quedas no ano anterior & & & & & $0,000^{* *}$ \\
\hline Não & $70,8 \%$ & $75,1 \%$ & $70,8 \%$ & $43,1 \%$ & \\
\hline Sim & $29,2 \%$ & $24,9 \%$ & $29,2 \%$ & $56,9 \%$ & \\
\hline $\mathrm{N}$ de quedas & $1,20( \pm 0,9)$ & $0,31( \pm 1,25)$ & $1,31( \pm 0,93)$ & $2,55( \pm 2,60)$ & $0,009^{* * *}$ \\
\hline \multicolumn{6}{|l|}{ Escores de Capacidade Funcional } \\
\hline ABVD & $0,14( \pm 0,42)$ & $0,09( \pm 0,30)$ & $0,12( \pm 0,35)$ & $0,36( \pm 0,80)$ & $0,000^{*}$ \\
\hline AIVD & $19,70( \pm 2,19)$ & $20,37( \pm 1,27)$ & $19,71( \pm 2,04)$ & $17,37( \pm 3,39)$ & $0,000^{*}$ \\
\hline AAVD & $3,01( \pm 2,03)$ & $2,70( \pm 1,79)$ & $3,06( \pm 2,07)$ & $3,86( \pm 2,38)$ & $0,000^{*}$ \\
\hline Escores FES-I* & $26,24( \pm 9,69)$ & $23,18( \pm 6,99)$ & $26,42( \pm 9,44)$ & $33,46( \pm 12,37)$ & $0,000^{*}$ \\
\hline Escores GDS ${ }^{\star}$ & $7,49( \pm 1,94)$ & $7,13( \pm 1,77)$ & $7,59( \pm 1,98)$ & $8,18( \pm 2,09)$ & $0,000^{*}$ \\
\hline
\end{tabular}

"significativo ( $\mathrm{p}<0,05)$, pelo teste ANOVA; " significativo $(\mathrm{p}<0,05)$, pelo teste Qui-Quadrado; * "** diferença entre os grupos frágil e não frágil $(\mathrm{p}=0,008) . "$ Falls Efficacy Scale - Brasil (FESI - Brasil). "Geriatric Depression Scale (GDS). 
Tabela 2. Distribuição de comorbidades avaliadas por autorrelato de acordo com os perfis de fragilidade frágil, pré-frágil e não frágil em idosos comunitários brasileiros avaliados pela Rede Fibra.

\begin{tabular}{|c|c|c|c|c|c|}
\hline Comorbidade $^{*}$ & $\begin{array}{c}\text { Amostra total } \\
\mathbf{n}=5532 \\
\% \text { positivo }\end{array}$ & $\begin{array}{c}\text { Não-frágeis } \\
\text { n= 2094 } \\
(37,8 \%) \\
\% \text { positivo }\end{array}$ & $\begin{array}{c}\text { Pré-frágeis } \\
\mathbf{n}=2821 \\
(51 \%) \\
\% \text { positivo }\end{array}$ & $\begin{array}{c}\text { Frágeis } \\
\mathrm{n}=617 \\
(11,2 \%) \\
\% \text { positivo }\end{array}$ & $\mathrm{p}$ \\
\hline Doença Cardíaca - angina, infarto & $18,4 \%$ & $15,9 \%$ & $19,5 \%$ & $22,1 \%$ & $0,000^{* *}$ \\
\hline Hipertensão Arterial Sistêmica (HAS) & $58,6 \%$ & $57,7 \%$ & $58,7 \%$ & $60,6 \%$ & 0,438 \\
\hline Acidente Vascular Encefálico (AVE) & $6,0 \%$ & $3,9 \%$ & $6,0 \%$ & $12,8 \%$ & $0,000^{* *}$ \\
\hline Diabetes Mellitus (DM) & $19,7 \%$ & $15,8 \%$ & $21,2 \%$ & $26,6 \%$ & $0,000^{* *}$ \\
\hline Câncer & $4,5 \%$ & $4,5 \%$ & $4,8 \%$ & $3,6 \%$ & 0,415 \\
\hline Osteoartrite (OA) & $32,2 \%$ & $30,5 \%$ & $32,6 \%$ & $35,9 \%$ & $0,031^{* *}$ \\
\hline Doença de pulmão & $7,8 \%$ & $6,9 \%$ & $8,2 \%$ & $9,4 \%$ & 0,065 \\
\hline Depressão & $16,5 \%$ & $13,6 \%$ & $18,2 \%$ & $18,8 \%$ & $0,000^{* *}$ \\
\hline Osteoporose & $22,4 \%$ & $20,9 \%$ & $22,6 \%$ & $26,7 \%$ & $0,010^{* *}$ \\
\hline
\end{tabular}

*baseado na pergunta "No último ano, algum médico já disse que o(a) senhor(a) tem os seguintes problemas de saúde?"

** significativo $(\mathrm{p}<0,05)$, pelo teste Qui-Quadrado.

Tabela 3. Frequência dos itens componentes do Fenótipo de Fragilidade na população idosa brasileira avaliada pela Rede Fibra.

\begin{tabular}{|c|c|c|c|}
\hline Itens de fragilidade & $\begin{array}{l}\text { Amostra } \\
\text { total } \\
n=5532\end{array}$ & $\begin{array}{c}\text { Pré-frágeis } \\
n=2821\end{array}$ & $\begin{array}{l}\text { Frágeis } \\
\mathrm{n}=617\end{array}$ \\
\hline Perda de peso & $17,3 \%$ & $23,4 \%$ & $45,9 \%$ \\
\hline Exaustão & $19,7 \%$ & $25,7 \%$ & $56,1 \%$ \\
\hline Nível de atividade física & $27,5 \%$ & $36,7 \%$ & $78,2 \%$ \\
\hline Força muscular & $20,6 \%$ & $24,4 \%$ & $70,2 \%$ \\
\hline Lentidão da marcha & $20,9 \%$ & $23,8 \%$ & $76,7 \%$ \\
\hline
\end{tabular}

O item exaustão, quando positivo, representou maior chance $(\mathrm{OR}=4,19$, IC95\%3,47-5,06, $\mathrm{p}$ $<0,001)$ para fragilidade em idosos pré-frágeis (Tabela 5).

Em modelo com todos os itens em conjunto, a capacidade de explicação foi de 99,6\% da fragilidade, mas os valores de odds ratio (OR) individual de cada item foi muito pequeno. Os itens em conjunto, quando positivos, apresentaram OR de alta magnitude para o desenvolvimento da fragilidade, sendo os maiores o nível de atividade física $(\mathrm{OR}=673,74$, IC95\%314,19-1444,75, $\mathrm{p}<0,000)$ e a força muscular $(\mathrm{OR}=513,37$, IC95\%261,00-1009,74, p < 0,000) (Tabela 5).

\section{Discussão}

O percentual de idosos frágeis, pré-frágeis e não frágeis encontrados na amostra foi semelhante ao de estudos internacionais ${ }^{2,19,20}$ e nacionais ${ }^{21,22}$, que também utilizaram o fenótipo de fragilidade para identificação da síndrome.

O perfil da amostra, de uma forma geral, foi semelhante ao encontrado por outros estudos, o que auxilia na comparação dos resultados. Em relação ao sexo e à idade, foi semelhante ao encontrado por Fried et al. ${ }^{2}$. Em relação à ocorrência de quedas, os resultados se assemelham aos de Ensrud et al. ${ }^{23}$, em que idosos frágeis tiveram mais chance de sofrer uma queda e as fraturas decorrentes dela. O nível de capacidade funcional foi pior entre os idosos frágeis, como também aconteceu em outros estudos ${ }^{2,24}$. O medo de cair foi mais relatado pelos idosos considerados frágeis, bem como no estudo de Dias et al. ${ }^{25}$, no qual idosos que pontuaram positivo para o item exaustão e lentidão da marcha restringiram mais suas atividades devido à esta consequência da ocorrência de quedas ${ }^{25}$.

Assim como no estudo de Woods et al. $^{26}$, as comorbidades foram mais comuns em idosos frágeis. $\mathrm{O}$ estudo de Klein et al. ${ }^{27}$ encontrou diferenças entre os grupos para doença cardíaca e diabetes, e outro estudo encontrou a diabetes como doença capaz de predizer a piora da fragilidade $^{20}$. A depressão foi fortemente associada à fragilidade em estudo de revisão da literatura sobre o tema ${ }^{28}$. Um estudo nacional com ido- 
Tabela 4. Influência de cada item de fragilidade para desenvolvimento da síndrome da fragilidade em idosos pré-frágeis.

\begin{tabular}{lccccc}
\hline Item do fenótipo Idosos pré-frágeis & Referência & $\mathbf{R}^{2}$ & OR & IC95\% & $\mathbf{p}$ \\
\hline Perda de peso & Não & 0,19 & 2,76 & $2,30-3,31$ & $0,000^{*}$ \\
Exaustão & Não & 0,23 & 3,68 & $3,08-4,41$ & $0,000^{*}$ \\
Nível de atividade física & Não & 0,37 & 6,35 & $5,16-7,81$ & $0,000^{*}$ \\
Força muscular & Não & 0,28 & 7,31 & $6,02-8,86$ & $0,000^{*}$ \\
Lentidão na marcha & Não & 0,32 & 10,50 & $8,55-12,90$ & $0,000^{*}$ \\
\hline
\end{tabular}

"significativo $\mathrm{p}<0,05$. Categoria de referencia variável dependente: Frágil.

Tabela 5. Modelos com itens agregados para avaliação de fragilidade para idosos pré-frágeis.

\begin{tabular}{lccccc}
\hline \multicolumn{1}{c}{ Combinações } & Referência & $\mathbf{R}^{2}$ & OR & IC95\% & p \\
\hline 5 itens: & & 0,996 & & & \\
$\quad$ Exaustão & Não & & 442,05 & $219,00-892,25$ & $0,000^{*}$ \\
$\quad$ Perda de peso & Não & & 309,51 & $158,34-605,01$ & $0,000^{*}$ \\
$\quad$ Nível de atividade física & Não & & 673,74 & $314,19-1444,75$ & $0,000^{*}$ \\
$\quad$ Força muscular & Não & & 513,37 & $261,00-1009,74$ & $0,000^{*}$ \\
$\quad$ Lentidão na marcha & Não & & 436,59 & $226,37-842,08$ & $0,000^{*}$ \\
3 itens: & & 0,696 & & & \\
$\quad$ Nível atividade física & Não & & 13,45 & $10,09-17,92$ & $0,000^{*}$ \\
$\quad$ Força muscular & Não & & 18,11 & $13,65-24,02$ & $0,000^{*}$ \\
$\quad$ Lentidão na marcha & Não & & 16,56 & $12,62-21,72$ & $0,000^{*}$ \\
2 itens: & & 0,390 & & & \\
$\quad$ Exaustão & Não & & 4,19 & $3,47-5,06$ & $0,000^{*}$ \\
Perda de peso & Não & & 3,26 & $2,69-3,95$ & $0,000^{*}$ \\
\hline
\end{tabular}

*significativo $\mathrm{p}<0,05$. Categoria de referência variável dependente: Frágil.

sos com osteoartrite também encontrou que os frágeis eram mais deprimidos em relação aos pré-frágeis e aos não frágeis ${ }^{29}$. Desta forma, em relação às comorbidades, o presente estudo se assemelha a outros, evidenciando que, no Brasil, comorbidade e fragilidade são entidades relacionadas, que devem ser colocadas em destaque, dentro da atenção à saúde do idoso.

Os valores da prevalência dos itens de fragilidade variam muito entre os estudos com populações diferenciadas. Estudo em Taiwan encontrou os mesmos itens, nível de atividade física e lentidão na marcha, como os mais prevalentes na amostra $^{30}$, e outro observou que o nível de atividade física foi o que mais aumentou em acompanhamento da amostra ${ }^{8}$. Um estudo com a população brasileira encontrou a exaustão como item mais frequente, seguida pela lentidão da marcha ${ }^{10}$. Este estudo também era com idosos comunitários, porém somente da cidade de Belo
Horizonte, o que pode explicar a variação dos resultados em relação ao presente estudo ${ }^{10}$. Embora existam tais variações dentro da literatura e no presente estudo, os resultados têm em comum o destaque dos itens ligados à questão física, direcionando para uma maior atenção para a sarcopenia e para a prevenção da inatividade entre os idosos.

Em relação a cada modelo traçado para observação da contribuição de cada item isoladamente para o desenvolvimento da fragilidade, idosos pré-frágeis, quando positivos para lentidão da marcha, tiveram 10,50 mais chance de se tornarem frágeis. A velocidade de marcha é considerada importante indicativo de desfechos adversos físicos e cognitivos entre os idosos ${ }^{31}$, sendo que a velocidade inferior a $0,8 \mathrm{~m} / \mathrm{s}$ é preditiva de desfechos adversos para a saúde do idoso ${ }^{32}$. Dentro do fenótipo de fragilidade, tal item é o mais fortemente associado a incapacidade, institucio- 
nalização e quedas com lesão ${ }^{8}$. Para o desenvolvimento da fragilidade, o presente estudo corroborou o estudo de Romero-Ortuno et al..$^{33}$, no qual a lentidão da marcha também apresentou maior associação para desenvolvimento da síndrome. A medida da velocidade de marcha é simples e pode ser realizada no contexto dos serviços de saúde, destacando a importância de sua incorporação em uma avaliação geriátrica abrangente. A velocidade da marcha pode ser considerada ferramenta isolada para avaliação da fragilidade na prática clínica, por apresentar alto poder preditivo isolado e em combinação com outros itens, além de ser um teste rápido e de baixo custo ${ }^{1}$.

Neste mesmo estudo ${ }^{33}$, entretanto, a lentidão também teve maior poder explicativo da variação da variável dependente, ao passo que na presente pesquisa foi o item baixo nível de atividade física, apesar da diferença ter sido pouca entre eles. Os dois itens, segundo outros estudos, são capazes de predizer também outros efeitos adversos, como hospitalização e morte ${ }^{6,34}$.

A diminuição da força muscular foi o terceiro item que mais explicou a fragilidade entre os modelos individuais. Este item foi considerado o primeiro a se manifestar na instalação da préfragilidade ${ }^{35}$, sendo aquele com maior risco de se tornar positivo em idosos inicialmente não frágeis $^{36}$. Esta informação é diferente do que ocorre neste estudo, pois aqui a instalação da fragilidade foi observada à partir da pré-fragilidade. A força muscular é considerado a medida mais direta da sarcopenia ${ }^{37}$, que é uma das bases do tripé da fragilidade, juntamente com alterações imunológicas e neuroendócrinas ${ }^{2}$. Apesar de ter sido o segundo item mais frequente de fragilidade em alguns estudos ${ }^{7,31}$, e o terceiro mais frequente neste, não foi associado individualmente a nenhum desfecho adverso em outro ${ }^{8}$. Tais resultados apontam que a fraqueza muscular, embora seja uma medida indireta de sarcopenia importante, precisa estar em conjunto com os demais itens do fenótipo na determinação da fragilidade, não podendo ser usada isoladamente, como a lentidão da marcha.

A exaustão foi o quarto item mais prevalente e tem forte associação com sintomas depressivos, que por sua vez também é muito ligada à fragilidade $^{26}$. Dentro da determinação da fragilidade, fica atrás de outros itens na capacidade de predizer desfechos adversos ${ }^{8}$.

A perda de peso é o item que menos aumentou a chance de desenvolvimento da fragilidade entre os idosos pré-frágeis. A perda de peso informa sobre o estado nutricional do idoso, pois a redução de nutrientes faz parte do ciclo de fragilidade 2 . Foi um dos itens menos comuns no estudo de Drey et al. ${ }^{7}$ e ainda foi aquele com menor poder de explicação para o desenvolvimento da fragilidade ${ }^{33}$. Tais resultados corroboram os achados deste estudo. Os itens exaustão e perda de peso apresentaram as relações mais indiretas com a fragilidade, sendo os menos capazes de sua determinação, individualmente.

Em relação aos modelos combinados, o modelo que continha nível de atividade física, força muscular e lentidão na marcha foi capaz de explicar $69,6 \%$ da presença da fragilidade. Kulminski et al..$^{38}$ observaram que uma ferramenta para avaliação de fragilidade com 3 itens é capaz de predizer mortalidade a curto prazo. A combinação entre exaustão e perda de peso explicou menos a fragilidade, $39 \%$.

Tais resultados podem ser avaliados baseado naqueles encontrados por Xue et al. ${ }^{36}$. Tais autores observaram que os itens baixo nível de atividade física, força muscular e lentidão na marcha apresentaram maior risco relativo para desenvolvimento da fragilidade. Da mesma forma, a perda de peso e a exaustão não determinaram o desenvolvimento da fragilidade isoladamente, precisando estar sempre em combinação com outros itens para determinar o seu desenvolvimento ${ }^{36}$ Tal dado pode corroborar o menor poder explicativo destes itens combinados encontrado nesta pesquisa, precisando estar agregados a outros na determinação da fragilidade.

Os cinco itens combinados em um único modelo conseguiram explicar 99,4\% da fragilidade. Tal resultado pode sinalizar que os itens explicam melhor a fragilidade em conjunto e talvez não possam ser isolados na população brasileira. Pois, como ferramenta de rastreio e avaliação de fragilidade entre idosos, o Fenótipo de Fragilidade 2 deve ser sempre aplicado de forma completa, seja na pesquisa ou na prática clínica.

Gobbens et al. ${ }^{39}$ afirmam que existe um consenso dentro do qual os itens do fenótipo sempre deverão estar presentes em qualquer avaliação de fragilidade. O fenótipo de fragilidade é compreensível e multifatorial, e apresenta medida que pode ser aplicada na clínica e na pesquisa, e ainda representa o conceito mais citado e discutido na literatura especializada atual ${ }^{40}$.

O presente estudo é transversal e, dentro das possibilidades de tal desenho, não é possível inferir sobre o desenvolvimento da fragilidade, baseado na presença dos itens, ao longo do tempo, e se ter uma medida exata do risco da presença de cada item, em relação aos outros, ou à sua ausência. As medidas de associação são apresentadas 
em odds ratio, o que pode ser considerado uma limitação. Porém, a amostra muito numerosa e localizada em todas as regiões do Brasil garante ampla validade externa aos resultados, e sinaliza resultados importante na tentativa de incorporação da avaliação de fragilidade como rotina nos serviços de saúde.

Frente à escassez de evidências para a população brasileira a respeito do assunto, tanto em relação à aplicação do fenótipo de fragilidade como à influencia de cada item na determinação da fragilidade, os resultados do presente estudo sinalizam que é importante a aplicação de todos os itens. E ainda, identifica que deve ser dada atenção na abordagem daqueles idosos que pontuam positivamente para os itens lentidão na marcha, nível de atividade física e força muscular, uma vez que apresentam maior risco para se tornarem frágeis e maior susceptibilidade a desfechos adversos associados à fragilidade.

\section{Colaboradores}

SLA Silva participou do delineamento dos objetivos da pesquisa e formulação das hipóteses, análises dos dados, redação do manuscrito final. AL Neri, E Ferrioli e RA Lourenço participaram do delineamento, elaboração e coordenação do estudo da Rede Fibra nacional, revisão do manuscrito final. RC Dias participou delineamento dos objetivos desta pesquisa e formulação das hipóteses; delineamento, elaboração e coordenação do estudo da Rede Fibra nacional; revisão do manuscrito final. 


\section{Referências}

1. Van Kan A, Rolland Y, Bergman H, Morley JE, Kritvhevsky SB, Vellas B. Fralty assessment of older people in clinical practice expert opinion of a geriatric advisory panel. J Nutr Health 2008; 12(1):29-37.

2. Fried LP, Tangen CM, Walston J, Newman AB, Hirsch C, Gottdiener J, Seeman T, Tracy R, Kop WJ, Burke G, McBurnie MA; Cardiovascular Health Study Collaborative Research Group. Frailty in older adults: evidence for a phenotype. J Gerontol A Biol Sci Med Sci 2001; 56(3):M146-M156.

3. Walston J, Hadley EC, Ferrucci L, Guralnik JM, Newman AB, Studenski SA, Ershler WB, Harris T, Fried LP. Research agenda for frailty in older adults: toward a better understanding of physiology and etiology: summary from the American Geriatrics Society/National Institute on Aging Research Conference on Frailty in Older Adults. J Am Geriatr Soc 2006; 54(6):991-1001.

4. Gill TM, Gahbauer EA, Allore HG, Han L. Transitions between frailty states among community-living older persons. Arch Intern Med 2006; 166(4):418-423.

5. Ottenbacher KJ, Ostir GV, Peek MK, Snih SA, Raji MA, Markides KS. Frailty in older Mexican Americans. J Am Geriatr Soc 2005; 53(9):1524-1531.

6. Freiheit EA, Hogan DB, Strain LA, Schmaltz HN, Patten SB, Eliasziw M, Maxwell CJ. Operationalizing frailty among older residents of assisted living facilities. BMC Geriatr 2011; 11:23.

7. Drey M, Pfeifer K, Sieber CC, Bauer JM. The fried frailty criteria as inclusion criteria for a randomized controlled trial: personal experience and literature review. Gerontology 2011; 57(1):11-18.

8. Rothman MD, Leo-Summers L, Gill TM. Prognostic significance of potential frailty criteria. J Am Geriatr Soc 2008; 56(12):2211-2116.

9. Jardim CSF, Tomaz SAG, Silva VG, Silva SLA, Dias RC. Fenotipo de Fragilidade: quais os itens são mais frequentes em um grupo de idosos de Belo Horizonte. Geriatria \& Gerontologia 2013; 6(3):237-245.

10. Silva SLA, Silva VG, Pereira LSM, Dias JMD, Dias RC. Comparação entre diferentes pontos de corte na classificação do perfil de fragilidade. Geriatria \& Gerontologia 2011; 5(3):130-135.

11. Brucki SMD, Nitrini R, Caramelli P, Bertolocci PHL, Okamoto IH. Sugestões para o uso do Mini-Exame do Estado Mental no Brasil. Arq. Neuropsiq. 2003; 61(B):777-781.

12. Lino VT, Pereira SR, Camacho LA, Ribeiro Filho ST. Cross-cultural adaptation of the Independence in Activities of Daily Living Index (Katz Index). Cad Saude Publica 2008; 24(1):103-112.

13. Lawton MP, Brody EM. Assessment of older people: self-maintaning and instrumental activities of daily living. Gerontologist 1969; 9(3):179-186.

14. Pincus T. A Multidimensional Health Assessment Questionnaire (MDHAQ) for All Patients with Rheumatic Diseases to Complete at All Visits in Standard Clinical Care. Bull NYU Hosp Jt Dis 2007; 65(2):150160.

15. Camargos FF, Dias RC, Dias JM, Freire MT. Cross-cultural adaptation and evaluation of the psychometric properties of the Falls Efficacy Scale-International Among Elderly Brazilians (FES-I-BRAZIL). Bras J Phys Ter 2010; 14(3):237-243.
16. Almeida OP, Almeida SL. Confiabilidade da versão brasileira da escala de depressão geriátrica (GDS) versão reduzida. Arq Neuropsiq 1999: 57(2-B):421-426.

17. Batistoni SST, Neri AL, Cupertino APFB. Validade da Escala de Depressão do Center For Epidemiological Studies entre idosos brasileiros. Arq. Neuropsiq 2007; 41(4):598-695.

18. Lustosa LP, Pereira DS, Dias RC, Britto RR, Parentoni AN, Pereira LSM. Tradução e adaptação transcultural do Minnesota Leisure Time Activities Questionnaire em idosos. Geriatria \& Gerontologia 2012; 5(2):57-65.

19. Bandeen-Roche K, Xue QL, Ferrucci L, Walston J, Guralnik JM, Chaves P, Zeger SL, Fried LP. Phenotype of frailty: characterization in the women's health and aging studies. J Gerontol A Biol Sci Med Sci 2006; 61(3):262-266

20. Espinoza SE, Jung I, Hazuda H. Frailty transitions in the San Antonio Longitudinal Study of Aging. J Am Geriatr Soc 2012; 60(4):652-660.

21. Silva SLA, Viana JU, Silva VG, Dias JMD, Pereira LSM, Dias RC. Influence of Frailty and Falls on Functional Capacity and Gait in Community-Dwelling Elderly Individuals. Topics Geriat Rehab 2012; 28(2):128-134.

22. Silva SLA, Vieira RA, Arantes P, Dias RC. Avaliação da Fragilidade, funcionalidade e medo de cair em idosos atendidos em um serviço ambulatorial de Geriatria e Gerontologia. Fisio Pesq 2009; 16(2):120-125.

23. Ensrud KE, Ewing SK, Taylor BC, Fink HA, Stone KL, Cauley JA, Tracy JK, Hochberg MC, Rodondi N, Cawthon PM; Study of Osteoporotic Fractures Research Group. Frailty and risk of falls, fracture, and mortality in older women: the study of osteoporotic fractures. $J$ Gerontol A Biol Sci Med Sci 2007; 62(7):744-751.

24. Boyd CM, Xue QL, Simpson CF, Guralnik JM, Fried LP. Frailty, hospitalization, and progression of disability in a cohort of disabled older women. Am J Med 2005; 118(11):1225-1231.

25. Dias RC, Freire MT, Santos EG, Vieira RA, Dias JM, Perracini MR. Characteristics associated with activity restriction induced by fear of falling in community-dwelling elderly. Bras J Phys Ter 2011; 15(5):406-413.

26. Woods NF, LaCroix AZ, Gray SL, Aragaki A, Cochrane BB, Brunner RL, Masaki K, Murray A, Newman AB; Women's Health Initiative. Frailty: emergence and consequences in women aged 65 and older in the Women's Health Initiative Observational Study. J Am Geriatr Soc 2005; 53(8):1321-1330.

27. Klein BE, Klein R, Knudtson MD, Lee KE. Frailty, morbidity and survival. Arch Gerontol Geriatr 2005; 41(2):141-149.

28. Levers MJ, Estabrooks CA, Ross Kerr JC. Factors contributing to frailty: literature review. J Adv Nurs 2006; 56(3):282-291.

29. Miguel RC, Dias RC, Dias JM, da Silva SL, Menicucci Filho PR, Ribeiro TM. Frailty syndrome in the community-dwelling elderly with osteoarthritis. Rev Bras Reumatol 2012; 52(3):331-347.

30. Lin CC, Li CI, Chang CK, Liu CS, Lin CH, Meng NH, Lee YD, Chen FN, Li TC. Reduced health-related quality of life in elders with frailty: a cross-sectional study of community-dwelling elders in Taiwan. PLoS One 2011; 6(7):e21841. 
31. Chang YW, Chen WL, Lin FG, Fang WH, Yen MY, Hsieh CC, Kao TW. Frailty and its impact on health-related quality of life: a cross-sectional study on elder community-dwelling preventive health service users. PLoS One 2012; 7(5):e38079.

32. Abellan van KG, Rolland Y, Andrieu S, Bauer J, Beauchet O, Bonnefoy M, Cesari M, Donini LM, Gillette Guyonnet S, Inzitari M, Nourhashemi F, Onder G, Ritz P, Salva A, Visser M, Vellas B. Gait speed at usual pace as a predictor of adverse outcomes in community-dwelling older people an International Academy on Nutrition and Aging (IANA) Task Force. J Nutr Health Aging 2009; 13(10):881-889.

33. Romero-Ortuno R, Walsh CD, Lawlor BA, Kenny RA. A frailty instrument for primary care: findings from the Survey of Health, Ageing and Retirement in Europe (SHARE). BMC Geriatr 2010; 10:57.

34. Purser JL, Kuchibhatla MN, Fillenbaum GG, Harding T, Peterson ED, Alexander KP. Identifying frailty in hospitalized older adults with significant coronary artery disease. J Am Geriatr Soc 2006; 54(11):1674-1681.

35. Xue QL. The frailty syndrome: definition and natural history. Clin Geriatr Med 2011; 27(1):1-15.

36. Xue QL, Bandeen-Roche K, Varadhan R, Zhou J, Fried LP. Initial manifestations of frailty criteria and the development of frailty phenotype in the Women's Health and Aging Study II. J Gerontol A Biol Sci Med Sci 2008; 63(9):984-990.

37. Silva TAA, Frisoli Junior A, Pinheiro MM, Szejnfeld VL. Sarcopenia Associada ao Envelhecimento: Aspectos Etiológicos e Opções Terapêuticas. Rev Bras Reumat 2006; 46(6):391-397.

38. Kulminski AM, Ukraintseva SV, Kulminskaya IV, Arbeev KG, Land K, Yashin AI. Cumulative deficits better characterize susceptibility to death in elderly people than phenotypic frailty: lessons from the Cardiovascular Health Study. J Am Geriatr Soc 2008; 56(5):898-903.

39. Gobbens RJJ, Luijkx KG, Wijnen-Sponselee MT, Schols JMGA. Towards an Integral Conceptual of Frailty. BMC Geriat 2010; 14(3):175-181.

40. Fairhall N, Langron C, Sherrington C, Lord SR, Kurrle SE, Lockwood K, Monaghan N, Aggar C, Gill L, Cameron ID. Treating frailty--a practical guide. $B M C \mathrm{Med}$ 2011; 9:83-90

Artigo apresentado em 19/08/2015

Aprovado em 24/11/2015

Versão final apresentada em 26/11/2015 